Case Report

Oopen Access

CrossMark

\title{
Successful treatment of fetal tachycardia by sotalol
}

\begin{abstract}
Fetal tachyarrhythmia is one of the main reasons of referral to fetal cardiology clinic, ${ }^{1}$ because it might be associated with different forms of complex congenital heart disease ${ }^{2,3}$ and also cause high antenatal mortality and morbidity due to heart failure. ${ }^{4,5}$ There are is different protocols of medical therapy with variable clinical results. ${ }^{6}$ We are reporting a successful management of 2 cases of fetal tachycardia with heart failure signs who responded dramatically to oral Satolol.
\end{abstract}

Volume 9 Issue 3 - 2017

\author{
Mahmoud AISoufi \\ Consultant Pediatric Cardiologist, UAE
}

Correspondence: Mahmoud AlSoufi, Consultant Pediatric Cardiologist, Al, Jalila Hospita, Dubail, UAE, Tel 97I553405476, Email dr.m.soufi@hotmail.com

Received: June 26, 2017 | Published: July 18, 2017

\section{Case (I)}

$30 y e a r$ olds lady with 28 weeks gestational, normal pregnancy without risk factors. She was referred to fetal cardiology clinic from Gynaecology clinic due to fetal tachyarrhythmia with moderate amount of ascites and plural pleural effusion. The mother was investigated to rule out the secondary causes of tachyarrhythmia like hyperthyroidism, anemia, fever, electrolyte imbalance and etc which were all normal.

Fetal echocardiography was done at 29 weeks gestational age which showed normal fetal heart anatomy, tachycardia with fetal heart rate 250-290beats/ min, impaired cardiac function with moderate amount of plural effusion and ascites (Figure $1 \& 2$ ). We decided to start her on oral satolol as a trial to control the fetal tachyarrhythmia, so we sent the mother to adult cardiology clinic for routine check up before starting the medicine (Electrocardiogram, Echocardiogram, blood pressure, etc). We started her on low dose initially, $80 \mathrm{mg}$ orally twice daily and she was kept as inpatient under observation.

The patient tolerated the medicine very well and fetal heart rate check up was done daily for 3 days which showed no response in the heart rate so we increased the dose to $80 \mathrm{mg}$ three times daily orally and we kept her under observation every 3 days as outpatient (ECG for the mother and fetal heart rate monitoring), again no response in fetal heart rate with same amount of ascites and plural effusion.

After that we increased the dose to $160 \mathrm{mg}$ twice daily and after 3 days from this dose the mother remains stable without any change in her ECG (QTc interval) and blood pressure and the fetal heart rate reduced dramatically to $140-150 / \mathrm{min}$. Patient was kept in same dose of Satolol (160mg twice daily) and regular weekly follow up in fetal cardiology clinic.

After one week (31weeks gestational age), the fetal heart rate remains stable $(140-150 / \mathrm{min})$ and there was some improvement in the effusion and ascites amount. Patients continued on the same dose and the fetal heart rate remains stable with significant reduction in the effusion and ascites. At 36 gestational age the mother was delivered by normal vaginal delivery without complications and the baby had normal sinus rhythm by ECG and discharged home at the age of 3days after given appointment in pediatric cardiology for follow up.

\section{Case (2)}

32years lady, with 22 weeks gestational age ,she has diabetes mellitus on oral medication (metformin) she was referred to fetal cardiology clinic due to abnormal fetal heart beats for evaluation and management. Fetal echocardiography was done and reviled irregular fetal tachyarrhythmia with fetal heart rate about 260-270/ min, impaired cardiac functions, mild plural effusion and ascites with trivial pericardial effusion (Figure 3).

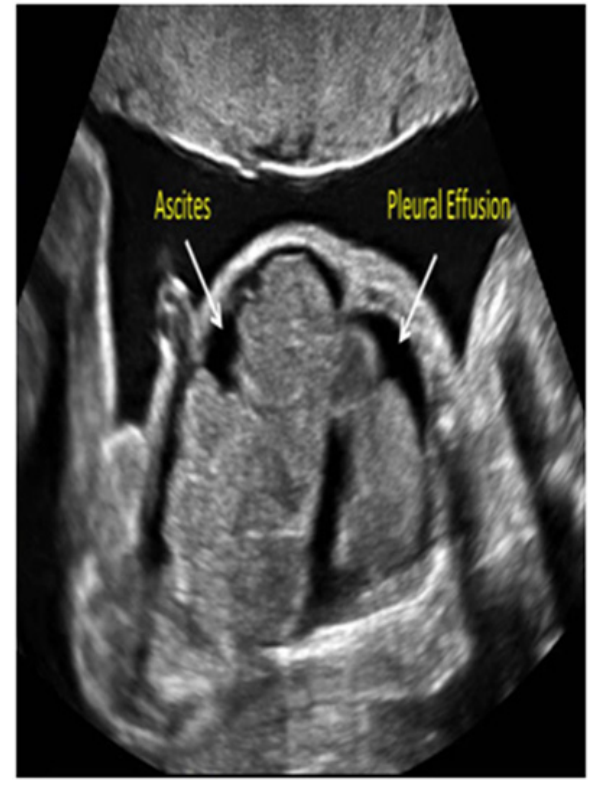

Figure I Ascitis and pleural effusion.

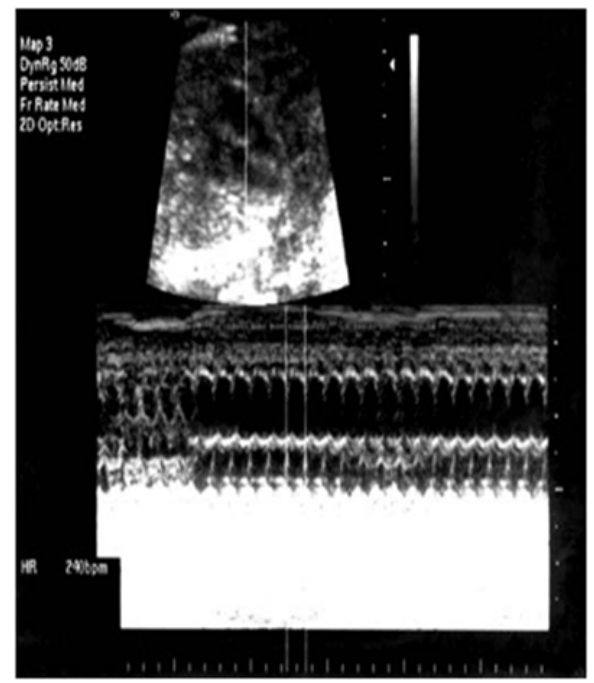

Figure 2 Fetal tachyarrhythmia. 


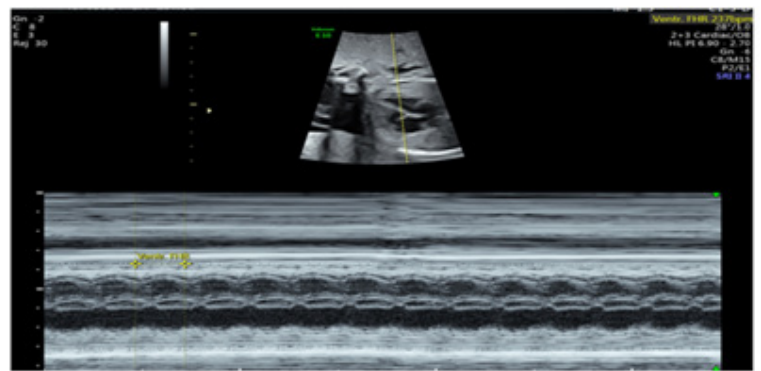

Figure 3 Fetal tachycardia with impaired function.

Patient was screened also to role out all secondary causes of fetal tachyarrhythmia (hyperthyroidism, fever, anemia, dehydration.....etc) and all were normal. She was admitted in the hospital and started on Sotalol $60 \mathrm{mg}$ twice daily orally after basic cardiac screening by adult cardiologist.

After 2days, she remain stable with normal vital signs and ECG but the fetal heart rate remains the same $(250-270 / \mathrm{min}$, so that we increased the dose to $160 \mathrm{mg}$ twice daily orally. Three days later the fetal screening showed slower heart rate (140-150/min) regular with same amount of plural effusion and ascites.

She was discharged home on same doses and given appointment after one week. One week later, fetal heart rate remains sinus, regular with normal cardiac functions and no pericardial effusion with less degree of plural effusion and ascites. After two weeks patient came to our clinic complaining of nausea, abdominal pain and mild vomiting ,her investigation showed signs of mild hepatitis with stable fetal heart rate, she stopped Sotalol herself and refused to continue and to take any medication and after 2 weeks she improved clinically with better liver function test and normal fetal heart.

\section{Discussion}

Fetal tachyarrhythmia is one of the leading cause of fetal hydrops and also it might cause fetal death due to heart and multi-organ failure. ${ }^{7}$

This cases might be associated with underline congenital heart diseases in 7\% like Ebstin Anomaly, transposition of great arteries, or with normal cardiac structure due to ectopic focus or accessory pathway. ${ }^{1,8}$

Fetal tachyarrhythmia can be of many types and and can be classified to the following:

a. Fetal sinus tachycardia: usually the rhythm is regular and it is a benign arrhythmia.

b. Fetal supraventricular tachycardia (SVT)

c. The commonest fetal tachyarrhythmia: accounts $60-90 \%$ of cases with ventricular rate of 240-280 beats per minute 4 and it is most likely associated with an accessory AV conduction pathway.

d. Fetal atrial flutter

e. Second most common fetal tachyarrhythmia 7: occur in 20 $\%$ of cases, with atrial rate of $300-600 \mathrm{bpm}$ but has a variable ventricular rate due to the frequent presence of an $\mathrm{AV}$ conduction block.

f. Fetal atrial fibrillation and fetal ventricular tachycardia: very rare condition.
Regarding the treatment of fetal tachyarrhythmia,

i. Usually we can control the arrhythmia by giving some medication to the mother, which passes through the placenta to the baby.

ii. Digoxin was used in multi cases because it is safety and efficacy but we have to give the mother high dose to reach the therapeutic serum level after few days and in case of hydrops the transmission of Digoxin will be affected and it will not be affective.

iii. Other anti arrhythmic drugs was used recently instead of digoxin as a first line for the treatment of fetal SVT like Flecanide and sotalol successfully especially with refractory SVT or SVT with signs of heart failure.

iv. Our 2 cases were treated successfully by small dose then moderate dose of Sotalol and they responded very well so that fetal heart reduced to normal fetal rate and the ascites and effusion disappeared and baby was born without any complications. ${ }^{9-11}$

\section{Conclusion}

Fetal arrhythmia should be detected early during pregnancy and treated as soon as possible to prevent its serious complications. The safe Antiarrhythmic drugs which cross the placenta like sotalol should be used with small doses and observe the mother and fetal heart, in case of no response, this dose can be increased gradually to achieve the target (normal fetal heart rate with no or minimal side effects on mother's health).

\section{Acknowledgements}

None.

\section{Conflicts of interest}

Author declares there are no conflicts of interest.

\section{Funding}

None.

\section{References}

1. Simpson JM, Sharland GK. Fetal tachycardias: management and outcome of 127 consecutive cases. Heart. 1998;79(6):576-581.

2. Lulić jurjević R, Podnar T, Vesel S. Diagnosis, clinical features, management, and post-natal follow-up of fetal tachycardias. Cardiol Young. 2009;19(5):486-493.

3. Brown DL. Sonographic assessment of fetal arrhythmias. AJR Am J Roentgenol. 1997;169(4):1029-1033.

4. Entezami M, Albig M, Knoll U. Ultrasound Diagnosis of Fetal Anomalies. Thieme. 2003.

5. Matta MJ, Cuneo BF. Doppler echocardiography for managing fetal cardiac arrhythmia. Clin Obstet Gynecol. 2010;53(4):899-914.

6. Fyfe DA, Meyer KB, Case CL. Sonographic assessment of fetal cardiac arrhythmias. Semin. Ultrasound CT MR. 1993;14(4):286-297.

7. Hornberger LK, Sahn DJ. Rhythm abnormalities of the fetus. Heart. 2007;93(10):1294-1300.

8. Rane HS, Purandare HM, Chakravarty A. Type and significance of fetal arrhythmias. Indian Heart J. 1996;48(1):40-44.

9. Reed KL. Fetal arrhythmias: etiology, diagnosis, pathophysiology, and treatment. Semin Perinatol. 1989;13(4):294-304.

10. Oudijk MA, Visser GH, Meijboom EJ. Fetal tachyarrhythmia--part I: Diagnosis. Indian Pacing Electrophysiol J. 2004;4(3):104-113.

11. Wren C. Mechanisms of fetal tachycardia. Heart. 1998;79(6):536-537. 